\title{
A Quadriparametric Model to Describe the Diversity of Waves Applied to Hormonal Data*
}

\author{
Saman Abdullah 1,2,3,4 Thomas Bouchard5; Amna Klich 1,2,3,4; Rene Leiva ${ }^{6,7}$; Cecilia \\ Pyper $^{8}$; Christophe Genolini9; Fabien Subtil 1,2,3,4; Jean Iwaz 1,2,3,4; René Ecochard 1,2,3,4 \\ ${ }^{1}$ Hospices Civils de Lyon, Service de Biostatistique-Bioinformatique, Lyon, France; \\ 2Université de Lyon, Lyon, France; \\ ${ }^{3}$ Université Lyon 1, Villeurbanne, France; \\ ${ }^{4}$ CNRS, UMR 5558, Laboratoire de Biométrie et Biologie Évolutive, Équipe Biostatistique-Santé, Villeurbanne, France; \\ ${ }^{5}$ Department of Family Medicine, University of Calgary, Calgary, Alberta, Canada; \\ ${ }^{6}$ Bruyère Research Institute, CT Lamont Primary Health Care Research Centre, Ottawa, Canada; \\ ${ }^{7}$ Department of Family Medicine, University of Ottawa, Ontario, Canada; \\ ${ }^{8}$ National Perinatal Epidemiology Unit, University of Oxford, Oxford, United Kingdom; \\ 9 INSERM, UMR 1027, Université Toulouse III, Toulouse, France
}

\section{Keywords}

Biomarker, wave, beta-binomial distribution, menstrual cycle, hormones

\section{Summary}

Background: Even in normally cycling women, hormone level shapes may widely vary between cycles and between women. Over decades, finding ways to characterize and compare cycle hormone waves was difficult and most solutions, in particular polynomials or splines, do not correspond to physiologically meaningful parameters.

Correspondence to:

Saman Abdullah

Service de Biostatistique-Bioinformatique, Hospices

Civils de Lyon

162, avenue Lacassagne - FR-69003 - Lyon - France

Phone: (+33) 611016696

E-mail: ext-s.abdulla@chu-lyon.fr

Supplementary material published on our website https://doi.org/10.3414/ME17-01-0102
Objective: We present an original concept to characterize most hormone waves with only two parameters.

Methods: The modelling attempt considered pregnanediol-3-alpha-glucuronide (PDG) and luteinising hormone (LH) levels in 266 cycles (with ultrasound-identified ovulation day) in 99 normally fertile women aged 18 to 45 . The study searched for a convenient wave description process and carried out an extended search for the best fitting density distribution. Results: The highly flexible beta-binomial distribution offered the best fit of most hormone

Methods Inf Med 2018; 57: 101-110 https://doi.org/10.3414/ME17-01-0102 received: September 28, 2017 accepted: November 28, 2017

Funding

This study was partially funded by Quidel Corporation, San Diego, CA, USA. waves and required only two readily available and understandable wave parameters: location and scale. In bell-shaped waves (e.g., PDG curves), early peaks may be fitted with a low location parameter and a low scale parameter; plateau shapes are obtained with higher scale parameters. I-shaped, J-shaped, and U-shaped waves (sometimes the shapes of LH curves) may be fitted with high scale parameter and, respectively, low, high, and medium location parameter. These location and scale parameters will be later correlated with feminine physiological events.

Conclusion: Our results demonstrate that, with unimodal waves, complex methods (e.g., functional mixed effects models using smoothing splines, second-order growth mixture models, or functional principal-component-based methods) may be avoided. The use, application, and, especially, result interpretation of four-parameter analyses might be advantageous within the context of feminine physiological events.

\section{Introduction}

Recently, several publications have renewed the interest in the assessment of individual hormone profiles along the menstrual cycle. This led to new analyses of these profiles in attempts to better understand the cycle [1].
During the menstrual cycle, hormone levels may vary dramatically between women and between successive cycles of the same woman. While simplifications are helpful for a basic physiological knowledge, it is interpreting individual hormone profiles that is required to diagnose and treat cycle abnormalities. Indeed, up to recently, the description of hormone curves have fo- cused on averages and ignored profile diversity. The literature did not recourse yet to more complex and/or recent methods such as the functional principal-component-based methods [2], the functional data analysis using smoothing splines [3], or the second-order growth mixture model [4]. It could be interesting to test these methods. However, hormone profiles are 
frequently simpler than the complex curve forms these methods are able to deal with. Besides, complex or recent models would use or require a rather high number of parameters that one could not be able to interpret biologically.

In our view, hormone profiles are characterized by the occurrence of one or several waves. Each wave exhibits specific characteristics: i) Amplitude reflects the hormone concentration (e.g., follicular stimulating hormone (FSH) shows one peak during the early pre-ovulatory phase and another peak immediately before ovulation; the amplitudes of these two peaks vary between women and between cycles of a given woman [5, 6]); ii) Wavelength reflects the duration of the hormone change (e.g., the length of the luteinizing hormone (LH) peak may range from a single day to about a week $[7,8])$; iii) Timing relative to the other hormone waves (e.g., during the luteal phase, the onset of the progesterone plateau may occur immediately before or after ovulation $[9,10])$; iv) Profile, which may have a triangular, arch, or plateau form [1]. Describing a hormonal profile requires that each of these four characteristics be taken into account.

Different methods have been already proposed to describe wave characteristics within the context of longitudinal latent class analysis. The statistical methods used to describe biological marker profiles may be separated into three categories: 1) crude descriptions of the profile without any smoothing or interpolation (these descriptions are made within the context of classifying longitudinal data, referred to as partitional clustering, using non-parametric algorithms like k-means [11]); 2) interpolation, using a polynomial or a spline regression over time [12-15]; 3) profile identification through a comparison with a given geometrical form (triangle, rectangle, plateau, or arch) or growth function [16] (this shape identification was previously applied to two-dimensional images but not to curves or waves).

To sum up, most previously published methods in the field were unable to provide readily useful and easily understandable descriptions of the cycle hormonal waves. Actually, non-parametric methods and interpolations are not "friendly" methods be- cause they require a great number of parameters (usually more than six) most of which are devoid of physiological meaning. Ideally, much less parameters should be sought for to allow their correspondence with clear feminine physiological events.

In the field of hormone waves, standardization may provide insights into hormone dynamics. Regarding wave amplitude, the daily hormonal concentration has been sometimes expressed as a proportion of its maximum [17]; this will be referred to as "standardization on the Y scale". Regarding wavelength, a standardization of time may be used to map several hormone profiles and compare them over a given time period (e.g., a theoretical 28-day cycle duration); this will be referred to as "standardization on the X scale". Regarding relative timing, most published studies have chosen to change the origin of time and use the day of maximum concentration as the reference day. However, using this reference conveys no information on the early or late occurrence of the hormonal peak within the menstrual cycle (today, the reference and gold standard is the day of ovulation as determined by ultrasounds). This relative timing will be referred to as "position."

\section{Objective}

In the present article, we introduce a new method that describes hormonal waves as density distributions. After standardization on the $\mathrm{X}$ and $\mathrm{Y}$ scales, the method is shown able to reflect two main wave characteristics: position and profile.

\section{Methods}

\subsection{Participants and Data}

The participants were recruited between 1996 and 1997 from eight Fertility Awareness Clinics located in France, Italy, Germany, Belgium, and Spain. This is the largest dataset with ultrasound detection of the ovulation day and daily measurements of hormone levels: it includes data on 107 normally fertile women aged 18 to 45 observed over an average of three cycles per woman; that is, data on 326 cycles.
The exclusion criteria were: a consistent history of anovulatory cycles, sub-fertility or active hormonal treatment for sub-fertility in the past three months, use of hormonal contraception or hormonal replacement therapy in the past three months, and abnormal cycle lengths.

During each cycle, a daily measurement of four hormones was performed: FSH, LH, urinary estrone-3-glucuronide (E1G), and urinary pregnanediol-3-alpha-glucuronide (PDG). The creatinine concentration was used to adjust for urine dilution. In addition, the day of ovulation was estimated using serial ultrasound scans.

Other data collected included age, age at menarche, parity, past oral contraceptive use, and lifestyle habits such as smoking, diet, and physical activity (hours/week), sleep duration (hours/day), and stress levels (subjective assessment). Height and weight were measured and the body mass index (BMI) calculated.

Herein, we have limited the analysis to those cycles with ultrasound-identified ovulation day; i.e., 283 among the 326 cycles. The seventeen cycles with a luteal phase of more than 17 days were arbitrarily considered to be possible pregnancies, and excluded from this analysis. Thus, 266 cycles out of 326 (82\%) were used for this analysis, provided by 99 women out of 107 (93\%). In addition, the hormonal profiles described were limited to PDG and LH.

\subsection{Statistical Analysis}

\subsubsection{Describing Amplitude and Wavelength}

Amplitude (the hormone concentration at its maximum) and wavelength (the length of post-ovulatory phase) were calculated. These two parameters will be further analysed for their predictive potential by characteristics of the women or by other characteristics of the cycle.

Then, standardization on the $\mathrm{Y}$ scale (amplitude) was carried out: PDG and LH levels of each cycle were rescaled within the range of the cycle-specific minimum and maximum. The standardization on the $\mathrm{X}$ scale (wavelength) will be considered during the description of the wave using a density distribution. 


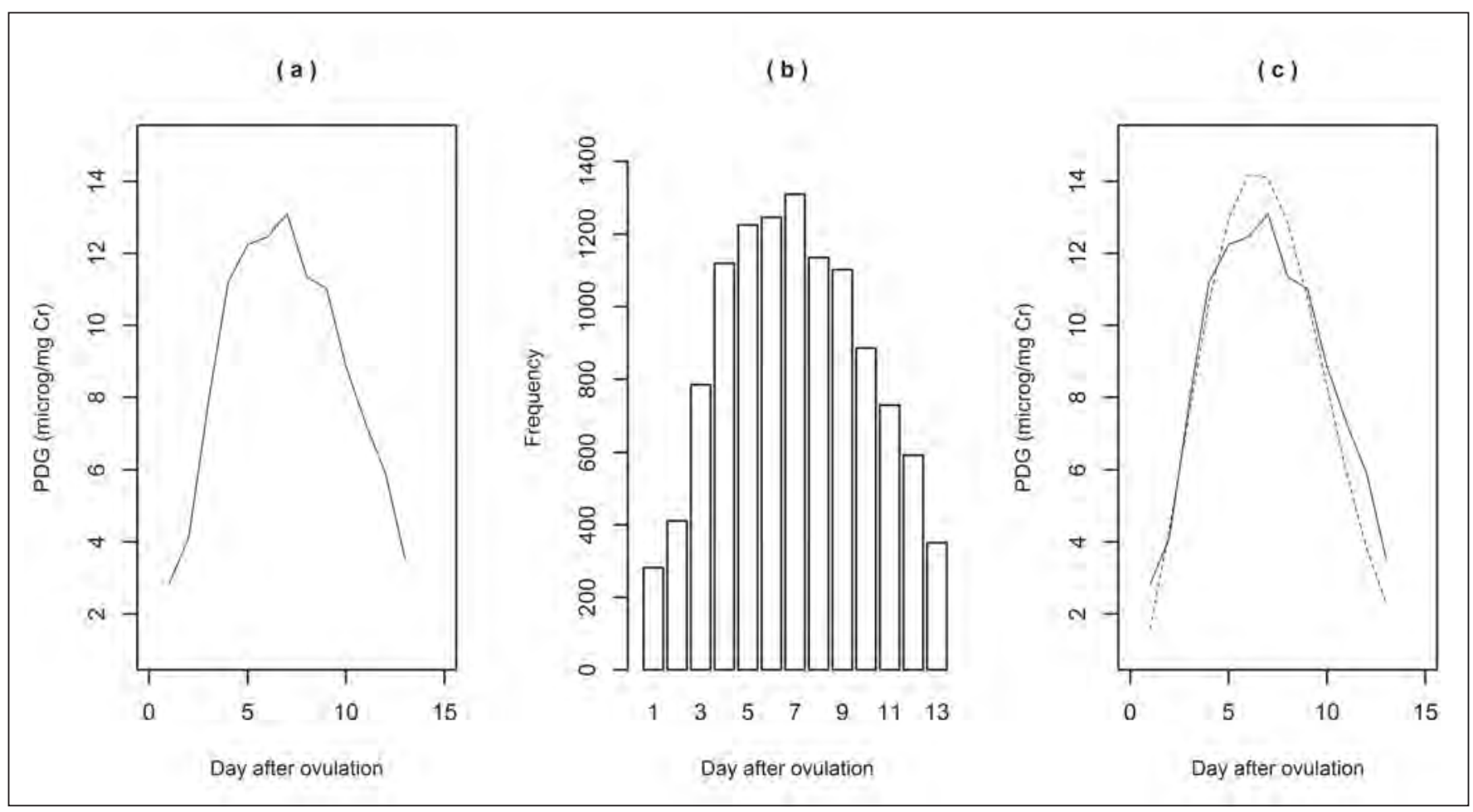

Figure 1 Applying the three-step process to describe a PDG hormonal curve. (a) The PDG hormonal curve. (b) The corresponding artificial dataset. (c) The observed (continuous line) vs. the fitted (dashed line) profile.

\subsubsection{Describing Hormonal Waves Using Density Distributions}

The profiles of the standardized hormonal wave were modelled as a density distribution. To describe waves using density distributions, there are two options:

1. The day is used as the explained variable and the day-specific hormone level as a frequency weight.

2. Preparing an artificial dataset whose density reflects perfectly the shape.

In practice, the first option was implemented for many known distributions in a generalized additive model for location, scale, and shape; namely gamlss library in R software.

When the first option is not implemented in the algorithm, a second option may be used through a wrapper ( $\triangleright$ Online Appendix 1).

- Figure 1 illustrates the above process of describing the profile of urinary PDG concentration during the luteal phase in one of the 266 cycles under study using the wrapper.
Note that, in this paper, the creation of an artificial dataset was avoided because the weight parameter is available in gamlss. In the process of the work, we tested both options to check whether our process is correct and obtained the same results.

\subsubsection{Fitting Hormonal Waves to Various Distributions}

Different distributions were applied: normal, Cauchy, Weibull... as well as binomial and beta-binomial.

The hormonal level being non-null at the beginning and the end of the wave, a two-side truncated version of these distributions was chosen for one of them, the normal distribution, to illustrate the benefit of truncation and compare with the betabinomial. The first and last days of the wave of each cycle were used as fixed truncation parameters.

The binomial distributions, especially the beta-binomial, have naturally non-null lower and upper densities: there was thus no need to use truncation for them. The first day of the wave was rescaled to zero and the length of the wave to the binomial denominator.

However, as mentioned below, discrete distributions would be more obvious.

- Table 1 lists the main distribution characteristics likely to be obtained upon changing some parameters. Some are symmetric (bell-shaped) and some asymmetric; some are right-skewed and some leftskewed. Moreover, with specific parameters, some are decreasing, increasing, or even U-shaped.

\subsubsection{Applying the Method to Describe PDG and LH Profiles}

This series of distributions was applied to the PDG and LH profiles of each cycle. The mean square of the residuals (MSE) was used as the loss function [18]. The best distribution was the one that minimized the loss function. A part of this loss function was reflecting daily oscillations; i.e., the measurement errors. Another part was due to the bias in the shape estimation. We chose MSE as low function because of its independence from the true underlying 
Table 1 Diversity of shapes likely to be described by various distributions upon using specific parameter values.

\begin{tabular}{|l|c|c|c|c|c|c|}
\hline & Shapes & & & & \\
\hline Distribution & $\begin{array}{l}\text { Symmetric } \\
\text { (bell-shaped) }\end{array}$ & $\begin{array}{l}\text { Right- } \\
\text { skewed }\end{array}$ & $\begin{array}{l}\text { Left- } \\
\text { skewed }\end{array}$ & Decreasing & Increasing & U-shaped \\
\hline Normal & $\sqrt{ }$ & & & & \\
\hline Cauchy & $\sqrt{ }$ & & & & \\
\hline Weibull & $\sqrt{ }$ & $\sqrt{ }$ & $\sqrt{ }$ & & \\
\hline Logistic & $\sqrt{ }$ & $\sqrt{ }$ & & & \\
\hline Gamma & & $\sqrt{ }$ & & $\sqrt{ }$ & \\
\hline Neg. binomial & & $\sqrt{ }$ & & & \\
\hline Log normal & $\sqrt{ }$ & $\sqrt{ }$ & & & \\
\hline Chi-squared & & $\sqrt{ }$ & & & \\
\hline Poisson & $\sqrt{ }$ & $\sqrt{ }$ & & $\sqrt{ }$ & \\
\hline Truncated normal & $\sqrt{ }$ & $\sqrt{ }$ & $\sqrt{ }$ & $\sqrt{ }$ & $\sqrt{ }$ \\
\hline B-binomial a & $\sqrt{ }$ & $\sqrt{ }$ & $\sqrt{ }$ & $\sqrt{ }$ & $\sqrt{ }$ \\
\hline
\end{tabular}

a See $>$ Online Appendix 3

distribution and to allow comparisons of fits obtained using the various density distributions. For MSE, the lowest value is the best.

The Durbin-Watson statistic was used as second criterion, the auto-correlation of residuals being a direct sign of bias in the shape estimation. The highest value of the Durbin-Watson statistic being the best, a low value is an effect of auto-correlation of the residuals; i.e., of the bias in the shape estimation.

We compared the speeds of convergence, the MSEs, and the Durbin-Watson statistics between the distributions and provide $>$ Table 2 in the case of two-side truncated normal distribution and beta-binomial distribution.

\subsubsection{Identification of Predictors of Wave Shape Using a Generalized Ad- ditive Model for Location and Scale}

A generalized additive model for location and scale was directly fitted to the dataset for each selected distribution. For illustration, we used the beta-binomial distribution and introduced factors as potential predictors of location and scale as well as a random intercept to take into account the hierarchical structure of the data (days within cycles and cycles within women). This model was originally described by Rigby and Stasinopoulos [19]. Here, we use it in a form limited to location and scale parameters. The model assumes independent observations $y_{i}$ for $i=1,2, \ldots, n$ with density functions $f\left(\boldsymbol{y}_{i} \mid \boldsymbol{\theta}^{i}\right)$ conditional on $\boldsymbol{\theta}^{i}=\left(\theta_{1 i}, \theta_{2 i}\right)=\left(\mu_{1 i}, \sigma_{i}\right)$. The latter two parameters are the location and scale parameters. For $k=1,2$, let $\boldsymbol{g}_{k}($.$) be known monotonic$ link functions relating the location and scale parameters to the explanatory variables by

$g_{k}\left(\boldsymbol{\theta}_{k}\right)=\boldsymbol{X}_{k} \boldsymbol{\beta}_{k}+\sum_{j=1}^{J_{k}} \boldsymbol{Z}_{j k} \boldsymbol{\gamma}_{j k}$

where $\boldsymbol{\theta}_{k}$ are vectors of length $n, X_{k}$ is the design matrix of predictors, $\boldsymbol{\beta}_{k}$ the vector of corresponding effects, $\boldsymbol{Z}_{j k}$ the design matrix of the hierarchy (days within cycles within women), and $\gamma_{j k}$ the vector of random effects. The link function applied for the location and scale parameters of the beta-binomial distribution were respectively the logit and the log functions.

The implementation of this model in $\mathrm{R}$ was presented by Stasinopoulos and Rigby [20].

Using weights as an artifice to fit the wave required dividing the weights by their sum at cycle level to maintain the correct number of degrees of freedom.

An abstract of the code is provided in -Online Appendix 2.

\subsection{Ethical Considerations}

In accordance with the French legislation in force at the time of this study, an observational study that did not change the routine management of patients did not need to be declared or submitted to the opinion of a research ethics board.

Table 2 Comparison of fitting process and performance between the truncated-normal (Notr) and the beta-binomial (BB) distributions.

\begin{tabular}{|l|l|l|l|l|l|l}
\hline & \multicolumn{2}{l}{ PDG (bell shaped) } & & \multicolumn{3}{l}{ LH (monotonically decreasing) } \\
\hline Criterion & BB & Notr & P value & BB & Notr & P value \\
\hline More than 20 iterations to reach convergence ${ }^{\text {a }}$ & $0 / 266(0 \%)$ & $37 / 266(14 \%)$ & $<0.01$ & $0 / 266(0 \%)$ & $242 / 266(91 \%)$ & $<0.01$ \\
\hline Mean Square Error $\left(10^{-4}\right)^{b}$ & $4.86(0.68)$ & $5.23(0.66)$ & $<0.01$ & $23.45(1.58)$ & $29.52(1.78)$ & $<0.01$ \\
\hline Durbin-Watson statistic $^{b}$ & $1.97(0.04)$ & $1.74(0.04)$ & $<0.01$ & $2.95(0.04)$ & $1.69(0.04)$ & $<0.01$ \\
\hline
\end{tabular}

a Proportion and chi-square test

${ }^{b}$ Mean (SEM) and Student t-test 


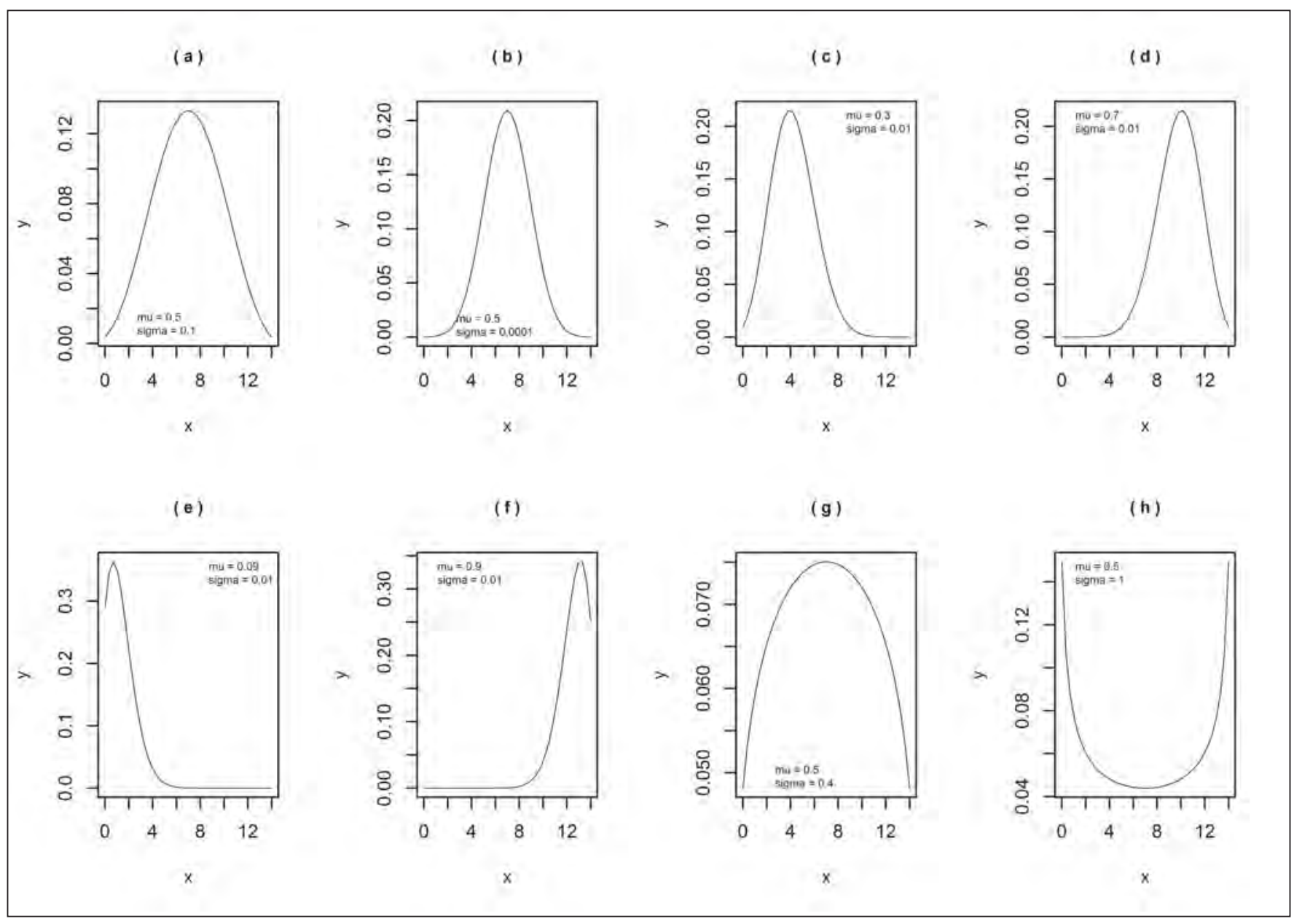

Figure 2 Shape diversity obtained with the beta-binomial distribution according to $\mu$ and $\sigma$ values. (a): Bell-shape. (b): Narrow peak. (c): Left-skew. (d): Right-skew. (e): Decrease. (f): Increase. (g): Arch. (h): U-shape.

\section{Results}

\subsection{Diversity of Shapes Identified by the Beta-binomial Distribution}

The beta-binomial distribution offers a high number of shapes upon changing only two parameters: $\mu$ and $\sigma$. $>$ Figure 2 illustrates some shapes obtained with various values of $\mu$ and $\sigma$. Figure 3 shows that a great diversity of PDG or LH hormonal profiles may be accommodated using the beta-binomial distribution.

The two-side truncated normal distribution provided very closely fitted waves. Nevertheless, this option had some limitations. As shown in Table 2, the convergence of the algorithm was much slower, the loss function was higher, and the Durbin-Watson statistic was in favour of more bias. The location and scale pa- rameters were quite similar to those of the beta-binomial for bell-shaped waves but differed dramatically for other shapes. In the latter case, these parameters were no more interpretable.

\subsubsection{Diversity of PDG and LH Profiles}

We applied the beta-binomial distribution to each PDG and LH profile of 266 cycles. We obtained for each the amplitude, the wavelength, parameter $\mu$, and parameter $\sigma$ of the beta-binomial distribution. We described the PDG waves and LH waves during the luteal phase using the beta-binomial distribution according to various $\mu$ and $\sigma$ values. For either PDG or LH, we considered three groups for $\mu$ values using the terciles $\left(33^{\text {rd }}\right.$ and $66^{\text {th }}$ percentiles of values dis- tribution) and three groups for $\sigma$ values. We obtained thus nine value supergroups. These nine groups were built to present all the waves in their diversity and show the power of the classification obtained using the betabinomial distribution.

- Figure 4 and $>$ Figure 5 show the typical profiles of PDG and LH profiles according to these supergroups of $\mu$ and $\sigma$ values.

For PDG profiles, Panels 4a, 4d, and $4 \mathrm{~g}$ of Figure 4, show symmetric or asymmetric bell-shaped profiles according to the value of $\mu$. The other panels show a plateau profile obtained either with higher values of $\sigma$ (vs. $4 \mathrm{a}, 4 \mathrm{~d}$, and $4 \mathrm{~g}$ ) and even increasing values of PDG toward the end of the menstrual cycle that correspond to the highest values of $\mu$ ( $\triangleright$ Figure $4 \mathrm{i}$ ). 


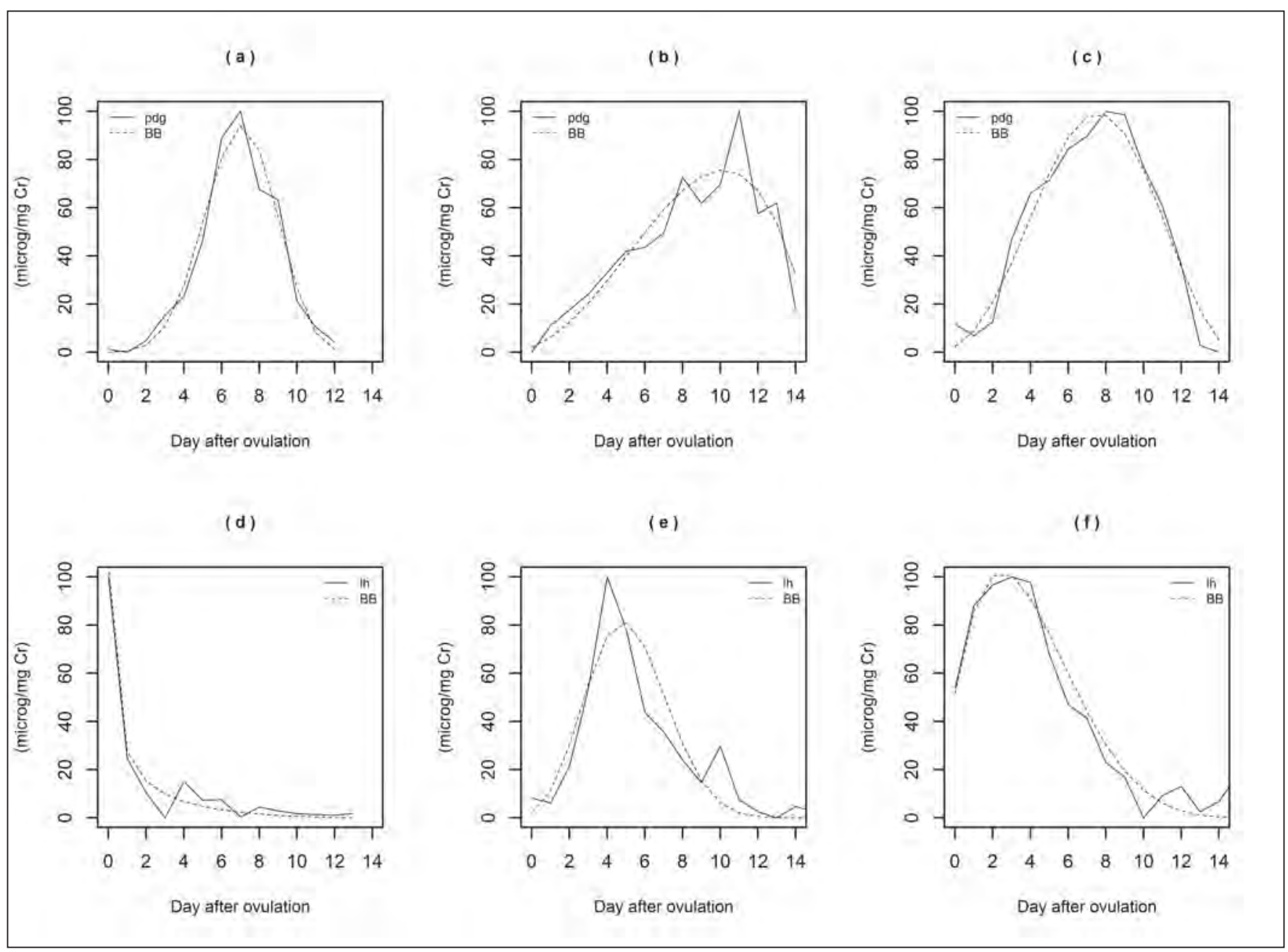

Figure 3 Examples of hormonal profiles starting at the ultrasound-determined day of ovulation (US-DO). (a) PDG - ID cycle: 1122 . (b) PDG - ID cycle: 5152. (c) PDG - ID cycle: 7033. (d) LH - ID cycle: 2103. (e) LH - ID cycle: 5103. (f) LH - ID cycle: 6113. The solid lines show the hormonal profiles and the dashed lines the fitted beta-binomial distribution.

For LH profiles, Panels $5 c$, 6f, and $5 \mathrm{i}$ of - Figure 5 show rapidly or more gradually decreasing curves obtained with high values of $\sigma$. The other panels show early (low $\mu$ ) or more delayed (higher $\mu$ ) peaks at the beginning of the luteal phase.

These graphs illustrate the high diversity of shapes fitted by the beta-binomial distribution.

\subsubsection{Identification of the Predictors of Wave Characteristics: Amplitude, Wavelength, and Wave Shape}

- Table 3 presents the result of a univariate regression of the characteristics of the wave on each predictor. The reason for showing only univariate results instead of multivariate results is that the causal path from most predictors might pass through others; for example, the maximum follicle size can be a result of age or BMI as well as a cause of the characteristics of the wave of post-ovulatory hormone level. A multivariate analysis would conceal the relationship between age or BMI and the characteristics of the wave.

All the predictors were previously standardized: they have mean zero and variance one to ease comparisons of effect sizes.

The wavelength appears to be significantly longer in older women, shorter after a long pre-ovulatory phase and in case of a small maximum follicle size, and associated with higher post-ovulatory $\mathrm{LH}$.

The amplitude was clearly and significantly lower in case of high BMI.
The location parameter was significantly higher in case of high post-ovulatory LH; i.e., a delayed increase of PDG after ovulation was associated with higher postovulatory LH.

A significantly higher scale parameter; i.e., a longer wave (up to plateau-like), was significantly associated with a higher PDG level on day 3 of the cycle, longer pre-ovulatory phase, and small maximum follicle size.

\section{Discussion}

We propose here a new mathematical method to describe the hormonal profiles observed during the menstrual cycle using a density distribution, with a preference for a two-side truncated normal distribution or 


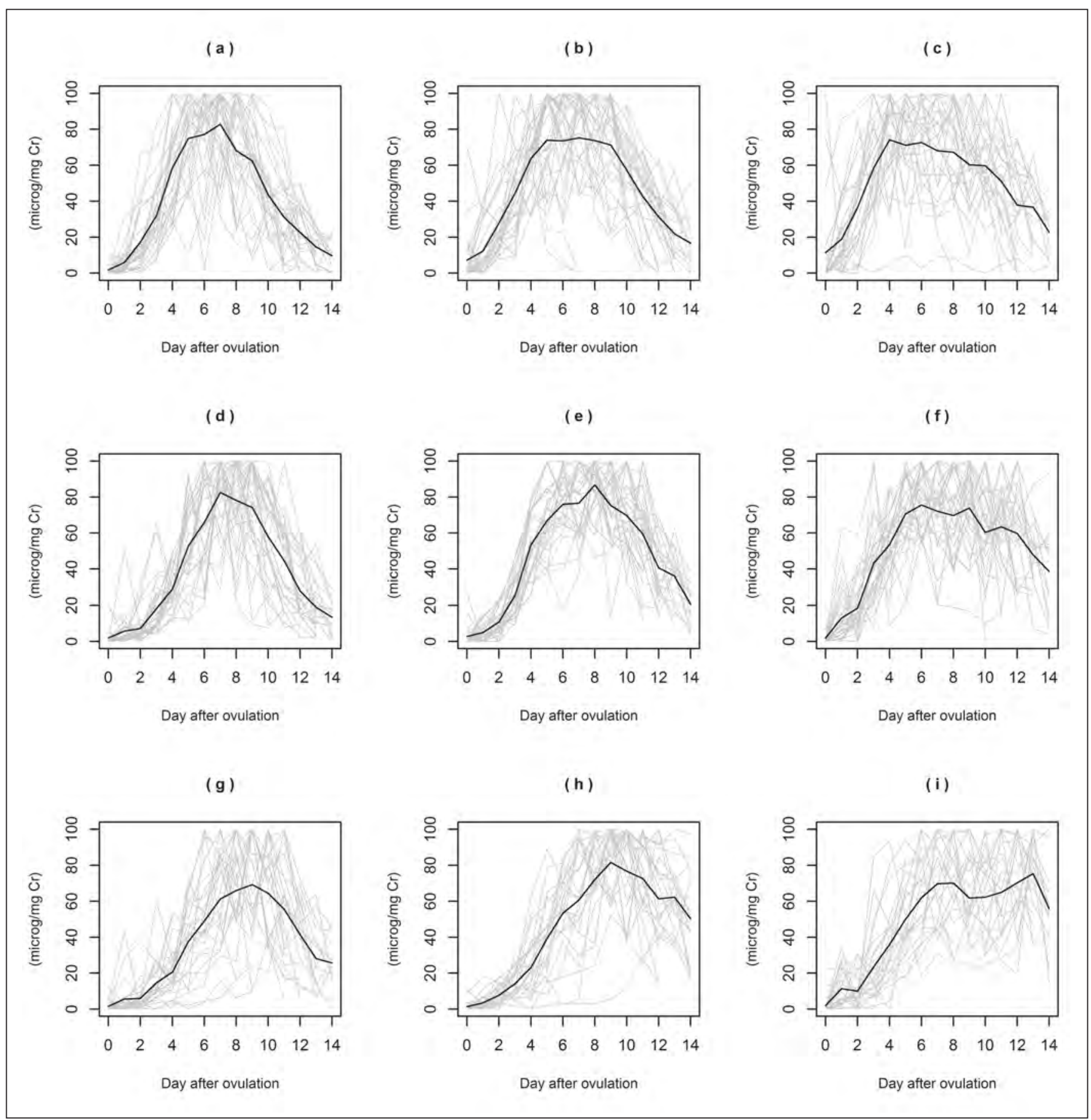

Figure 4 Beta-binomial-fitted PDG profiles according to $\mu$ (columns) and $\sigma$ (rows) terciles. (a) $\mu$ : $(0.35-0.55) ; \sigma:(0.01-0.12)$. (b) $\mu$ : $(0.35-0.55) ; \sigma$ : (0.12 - 0.18). (c) $\mu:(0.35-0.55) ; \sigma:(0.18-0.71)$. (d) $\mu$ : $(0.55-0.6) ; \sigma:(0.01-$ $0.13)$. (e) $\mu$ : $(0.55-0.6) ; \sigma:(0.13-0.17)$. (f) $\mu:(0.55-0.6) ; \sigma:(0.17-0.73)$. (g) $\mu$ : $(0.6-0.87) ; \sigma:(0.01-0.13)$. (h) $\mu:(0.6-0.87) ; \sigma:(0.13-0.19)$. (i) $\mu$ : $(0.6-0.87) ; \sigma:(0.19-0.53)$. The individual cycles are shown in thin lines and the group average in thick line. beta-binomial distribution, which proved to be the more appropriate in our context. With this new method, we were able to describe a wide variety of hormone profiles using only 4 parameters: amplitude, wavelength, position, and profile. The latter are estimated using a beta-binomial distribution with various $\mu$ and $\sigma$ values. One of the desired benefits of the density distribution (instead of spline) was to smooth the wave and avoid giving too much importance to the measurement error.
By adjusting the amplitude (Y-scale), using the length as a first fixed parameter of the beta-binomial distribution, and finally estimating different values for $\mu$ and $\sigma$ (the two parameters of the beta-binomial distribution), we were able to describe not 
(a)

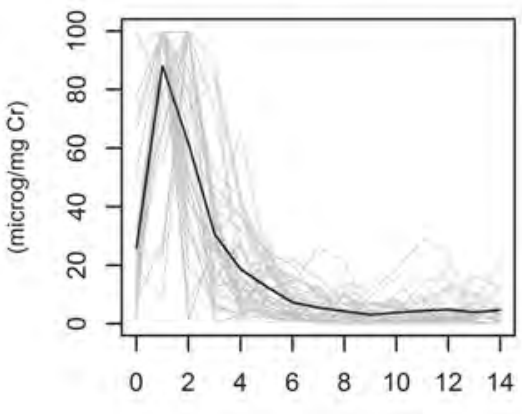

Day after ovulation

(d)

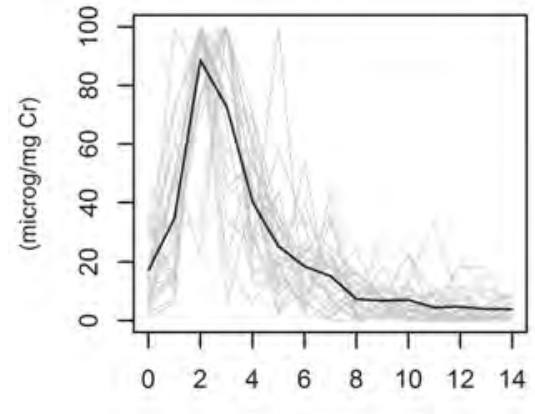

Day after ovulation

(g)

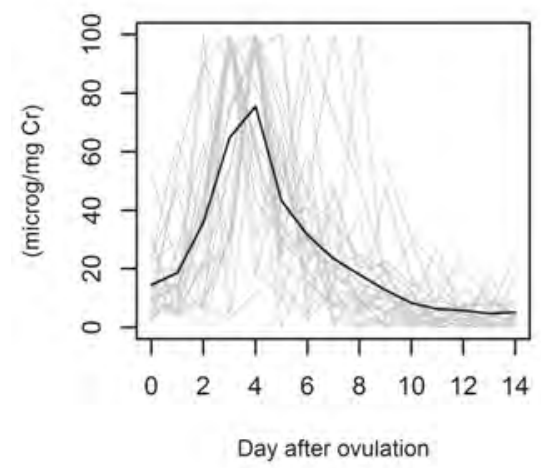

(b)

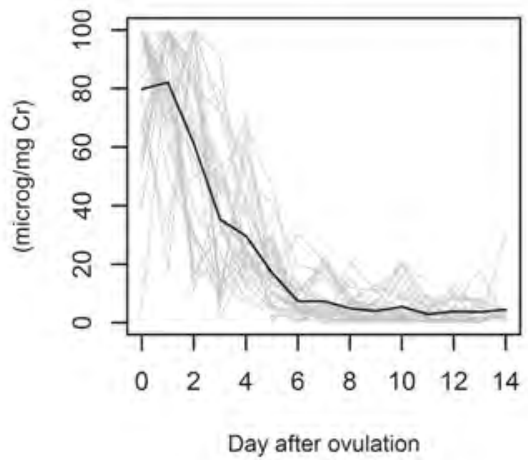

(e)

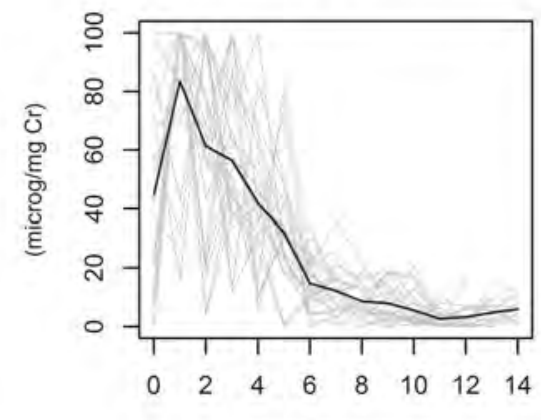

Day after ovulation

(h)

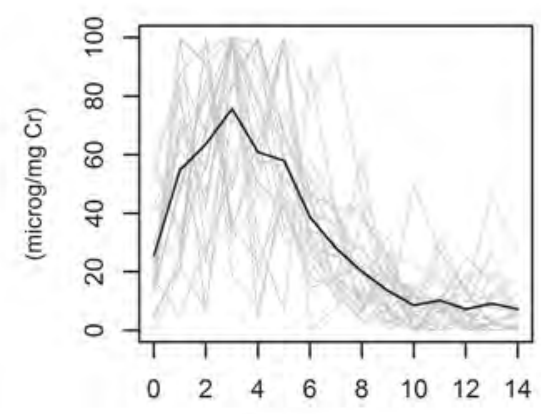

Day after ovulation (c)

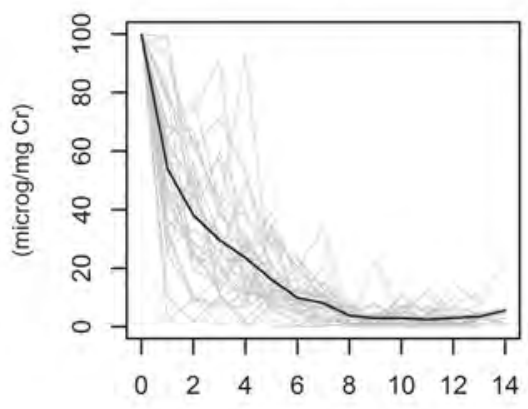

Day after ovulation

(f)

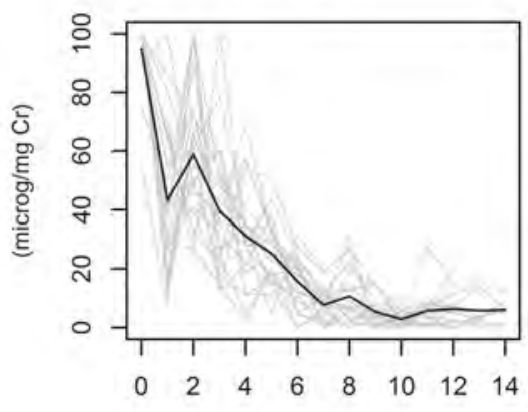

Day after ovulation

(i)

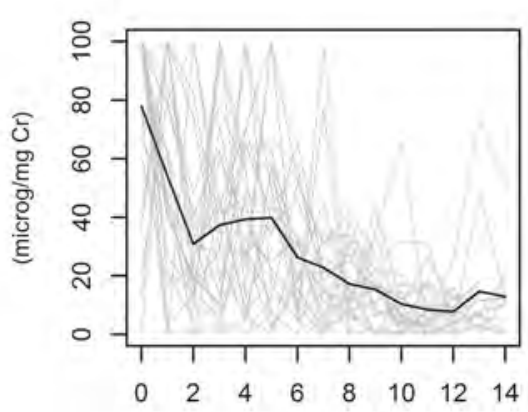

Day after ovulation
Figure 5 Beta-binomial-fitted LH profiles according to $\mu$ (columns) and $\sigma$ (rows) terciles. (a) $\mu$ : $(0.08-0.18) ; \sigma:(0.01-0.03)$. (b) $\mu$ : $(0.08-0.18) ; \sigma$ : $(0.03-0.14)$. (c) $\mu:(0.08-0.18) ; \sigma:(0.14-1)$. (d) $\mu:(0.18-0.24) ; \sigma:(0.01-$ $0.05)$. (e) $\mu:(0.18-0.24) ; \sigma:(0.05-0.16)$. (f) $\mu:(0.18-0.24) ; \sigma:(0.16-0.6)$. (g) $\mu$ : $(0.24-0.57) ; \sigma:(0.01-0.06)$. (h) $\mu:(0.24-0.57) ; \sigma:(0.06-0.19)$. (i) $\mu$ : $(0.24-0.57) ; \sigma:(0.19-1)$. The individual cycles are shown in thin lines and group average in thick line. only bell-shaped profiles, but also more complex profiles. This method allowed predicting directly the wave shape using a generalized additive model for location and scale of the beta-binomial distribution.
Several arguments led us to prefer the beta-binomial distribution. First, the betabinomial distribution is a common choice in analyses of proportion data with some form of added heterogeneity (See $>$ Appendix 3). Second, given that the time scale is discrete (i.e., one measurement per day), a discrete distribution was more suitable.

Third, the highly flexible beta-binomial distribution was able to describe most of the hormone waves (or curve shapes). We found that the simple and homogeneous (n, 
Table 3

Predictors of the four characteristics of the waves: amplitude, wavelength, location, and scale parameters $(\mu$ and $\sigma)$ of the beta-binomial distribution used to describe the PDG profile during the post-ovulatory phase (Univariate analysis, estimation over 266 cycles).

\begin{tabular}{ll|l|l|l}
\hline Level and predictor & Wavelength ${ }^{a}$ & Amplitude $^{\text {b }}$ & Location parameter & Scale parameter \\
\hline Woman level & & & & \\
\hline Age (years) & $0.254(0.101)^{*}$ & $-0.807(0.470)$ & $-0.015(0.017)$ & $-0.020(0.031)$ \\
\hline Body mass index & $-0.005(0.103)$ & $-1.185(0.467)^{*}$ & $-0.008(0.017)$ & $0.002(0.062)$ \\
\hline Cycle level & & & & \\
\hline PDG on day 3 of the cycle & $-0.141(0.102)$ & $0.196(0.472)$ & $0.001(0.019)$ & $0.104(0.029)^{*}$ \\
\hline Pre-ovulatory phase length & $-0.513(0.098)^{*}$ & $-0.531(0.471)$ & $-0.008(0.018)$ & $0.076(0.032)^{*}$ \\
\hline Maximum follicle size $<18 \mathrm{~mm}$ & $-0.859(0.310)^{*}$ & $-0.678(1.452)$ & $-0.082(0.054)$ & $0.206(0.093)^{*}$ \\
\hline Post-ovulatory LH level $(\mathrm{mIU} / \mathrm{mg} \mathrm{Cr})^{c}$ & $0.695(0.093)^{*}$ & $0.082(0.473)$ & $0.044(0.016)^{*}$ & $-0.011(0.029)$ \\
\hline
\end{tabular}

Results are expressed as value (SEM).

${ }^{a}$ Length of the post-ovulatory phase of the menstrual cycle.

${ }^{b}$ Maximum of PDG level.

${ }^{c}$ Creatinine-adjusted values.

${ }^{*} p<0.05$. $\mu$ ) binomial distribution offered a diversity of shapes: a bell-shape; a nearly normal shape when $\mu$ is close to 0.5 , an asymmetric distribution when $\mu>0.5$ (with a negative skew; i.e., a long tail to the left) or $<0.5$ (with a positive skew; i.e., a long tail to the right). When the probability was close to 1 , the density increased monotonically and, on the opposite, when the probability was close to 0 , the density decreased monotonically.

Fourth, the beta-binomial distribution allows introducing heterogeneity through parameter $\sigma$. This parameter increased the number of possible shapes: the kurtosis of this distribution may be high or low (even a plateau); and, with a very high $\sigma$, the distribution can even be I, J, or U-shaped with, respectively, low, high, or medium location parameter.

Fifth, the beta-binomial distribution reflects optimally the variability of hormone profiles because its parameters have direct interpretations: the peaks of the hormone curves seemed to shift to the right with increasing values of $\mu$ and show longer plateaus with increasing values of $\sigma$. This applied to PDG as well as to LH.

Sixth, the beta-binomial distribution adapts naturally to the wavelength, and this length was fixed through parameter $n$ before fitting the distribution to the data.

In this article, we described all profiles with a single type of distribution. An alternative approach would have been to select a distribution type for each cycle; however, this would have been at the price of greater complexity that would not allow for gen- eralizations according to groups of parameters among cycles with common characteristics. Obviously, in case of more complex profiles (e.g., bimodal shapes), it would be warranted to include two beta-binomial distributions.

The method may be applied to all data that translate into a unimodal distribution (most frequently, with a single peak). In case of two or more peaks, a mixture of distributions is needed -thus, much more than two parameters-, which requires much more complicated methods. The proposed method would then apply, for example, to the evolution of biomarkers in emergency units or to the evolution of serum insulin after a test for insulin resistance.

\section{Conclusion}

The present study has confirmed the diversity of hormonal waves in normally cycling women in terms of amplitude, wavelength, relative timing, and wave profile [1,7-9]. This diversity was observed not only between women but also between different cycles in a single woman. The results confirm that the beta-binomial distribution may help describing generalizable patterns of hormone variability.

The problem of hormone wave characterization has been long debated but is still unresolved. The method shown here offers a much less complex solution and allows much easier comparisons and interpretations. This method is more appropriate than polynomials or splines whenever the shape of the wave is to be correlated with other characteristics. In future studies, it will be worthwhile to confirm whether differences in the beta-binomial distribution characteristics reflect actually clinical parameters (e.g., age, body mass index) and whether the profiles of different hormones correlate with each other.

Furthermore, the concept and the method may be used in several other medical or scientific domains (physics, chemistry, etc.) whenever wave shapes have to be correlated with specific subject or object characteristics. The method applies ideally to curves that do not need a decomposition into two or more components (each with its own peak); i.e., the method does not apply to multimodal curves. For the latter curves, the above-cited more complicated methods (such as splines) would be more suitable.

\section{Acknowledgment}

The authors thank Drs Sophie Dubus, Anne Leduy, Isabelle Ecochard, Marie Grisard Capelle, Enriqueta Barranco, Michele Barbato, Sandro Girotto, and Marion Gimmler from the Fertility Awareness Clinics as well as all the women who took part in this study.

\section{Conflict of Interest}

The authors declare they have no conflicts of interest in relation to the present methodological work. 


\section{References}

1. Alliende ME. Mean versus individual hormonal profiles in the menstrual cycle. Fertil Steril 2002; 78(1): 90-96.

2. Besse P, Ramsay JO. Principal components analysis of sampled functions. Psychometrika 1986; 51(2): 285-311.

3. Guo W. Functional data analysis in longitudinal settings using smoothing splines. Stat Methods Med Res 2004; 13(1): 49-62.

4. Grimm KJ, Ram N. A Second-Order Growth Mixture Model for Developmental Research, Res Human Dev 2009; 6(2-3): 121-143.

5. Ecochard R, Guillerm A, Leiva R, Bouchard T, Direito A, Boehringer H. Characterization of follicle stimulating hormone profiles in normal ovulating women. Fertil Steril 2014; 102(1): 237-243.

6. Hall JE, Schoenfeld DA, Martin KA, Crowley WF Jr. Hypothalamic gonadotropin-releasing hormone secretion and follicle-stimulating hormone dynamics during the luteal-follicular transition. J Clin Endocrinol Metab 1992; 74(3): 600-607.

7. Park SJ, Goldsmith LT, Skurnick JH, Woitczuk A, Weiss G. Characteristics of the urinary luteinizing hormone surge in young ovulatory women. Fertil Steril 2007; 88(3): 684-690.

8. Direito A, Bailly S, Mariani A, Ecochard R. Relationships between the luteinizing hormone surge and other characteristics of the menstrual cycle in normally ovulating women. Fertil Steril 2013; 99(1): 279-285.

9. Blackwell LF, Vigil P, Alliende ME, Brown S, Festin $\mathrm{M}$, Cooke DG. Monitoring of ovarian activity by measurement of urinary excretion rates using the Ovarian Monitor, Part IV: the relationship of the pregnanediol glucuronide threshold to basal body temperature and cervical mucus as markers for the beginning of the post-ovulatory infertile period. Hum Reprod 2016; 31(2): 445-453.

10. Blackwell LF, Vigil P, Cooke DG, d'Arcangues C, Brown JB. Monitoring of ovarian activity by daily measurement of urinary excretion rates of oestrone glucuronide and pregnanediol glucuronide using the Ovarian Monitor, Part III: variability of normal menstrual cycle profiles. Hum Reprod 2013; 28(12): 3306-3315.

11. Genolini C, Falissard B. kml:k-means for longitudinal data. Comput Stat 2010; 25(2): 317-328.

12. Nagin DS, Odgers CL. Group-based trajectory modeling in clinical research. Ann Rev Clin Psychol 2010; 6: 109-138.

13. Muthén $B$. Latent variable analysis: growth mixture modeling and related techniques for longitudinal data. In: Handbook of Quantitative Methodology for the Social Sciences, ed. D. Kaplan. Newbury Park, CA: Sage; 2004: 345-368.

14. Nylund KI, Asparouhov T, Muthén BO. Deciding on the number of classes in latent class analysis and growth mixture modeling: a Monte Carlo simulation study. Struct Equ Model 2007; 14(4): 535-569.

15. Niu F, Zhou J, Le TH, Ma JZ. Testing the trajectory difference in a semi-parametric longitudinal model. Stat Methods Med Res 2017; 26(3): 1519-1531.

16. Nierop AF, Niklasson A, Holmgren A, Gelander L, Rosberg S, Albertsson-Wikland K. Modelling individual longitudinal human growth from fetal to adult life - QEPS I. J Theor Biol 2016; 406: 143-165.

17. Collins WP, Collins PO, Kilpatrick MJ, Manning PA, Pike JM, Tyler JP. The concentrations of urinary oestrone-3-glucuronide, LH and pregnanediol-3alpha-glucuronide as indices of ovarian function. Acta Endocrinol (Copenh) 1979; 90(2): 336-348.

18. Hennig C, Kutlukaya M. Some thoughts about the design of loss functions. Revstat Stat J 2007; 5(1): 19-39.

19. Rigby RA, Stasinopoulos DM. Generalized Additive Models for Location, Scale and Shape. Appl Stat 2005; 54(3): 507-554.

20. Stasinopoulos DM, Rigby RA. Generalized additive models for location scale and shape (GAMLSS) in R. J Stat Softw 2007; 23(7): 1-46. 